\title{
Fuzzy Integral Equations and Strong Fuzzy Henstock Integrals
}

\author{
Yabin Shao ${ }^{1,2}$ and Huanhuan Zhang ${ }^{1}$ \\ ${ }^{1}$ College of Mathematics and Computer Science, Northwest University for Nationalities, Lanzhou 730030, China \\ ${ }^{2}$ Department of Mathematics, Sichuan University, Chengdu 610065, China
}

Correspondence should be addressed to Huanhuan Zhang; sqhh1004@163.com

Received 29 April 2013; Revised 6 September 2013; Accepted 18 September 2013; Published 29 January 2014

Academic Editor: Márcia Federson

Copyright (c) 2014 Y. Shao and H. Zhang. This is an open access article distributed under the Creative Commons Attribution License, which permits unrestricted use, distribution, and reproduction in any medium, provided the original work is properly cited.

By using the strong fuzzy Henstock integral and its controlled convergence theorem, we generalized the existence theorems of solution for initial problems of fuzzy discontinuous integral equation.

\section{Introduction}

The fuzzy differential and integral equations are important part of the fuzzy analysis theory and they have the important value of theory and application in control theory.

The Cauchy problems for fuzzy differential equations have been studied by several authors [1-6] on the metric space $\left(E^{n}, D\right)$ of normal fuzzy convex set with the distance $D$ given by the maximum of the Hausdorff distance between the corresponding level sets. Seikkala in [7] defined the fuzzy derivative and then some generalizations of that have been investigated in $[8,9]$. Consequently, the fuzzy integral which is the same as that of Dubois and Prade in [10], by means of the extension principle of Zadeh, showed that the fuzzy initial value problem $x^{\prime}(t)=f(t, x(t)), x(0)=x_{0}$, has a unique fuzzy solution when $f$ satisfies the generalized Lipschitz condition which guarantees a unique solution of the deterministic initial value problem. Kaleva [1] studied the Cauchy problem of fuzzy differential equation and characterized those subsets of fuzzy sets in which the Peano theorem is valid. Park et al. in [11-14] have considered the existence of solution of fuzzy integral equation in Banach space. In 2002, Xue and Fu [15] established solutions to fuzzy differential equations with right-hand side functions satisfying Caratheodory conditions on a class of Lipschitz fuzzy sets.

However, there are discontinuous systems in which the right-hand side functions $f:[a, b] \times E^{n} \rightarrow E^{n}$ are not integrable in the sense of Kaleva [1] on certain intervals and their solutions are not absolute continuous functions. To illustrate, we consider the following example.
Example 1. Consider the following discontinuous system:

$$
\begin{gathered}
x^{\prime}(t)=h(t), \quad x(0)=\widetilde{A}, \\
g(t)= \begin{cases}2 t \sin \frac{1}{t^{2}}-\frac{2}{t} \cos \frac{1}{t^{2}}, & t \neq 0, \\
0, & t=0,\end{cases} \\
\widetilde{A}(s)= \begin{cases}s, & 0 \leq s \leq 1, \\
2-s, & 1<s \leq 2, \\
0, & \text { others, }\end{cases} \\
h(t)=\chi_{|g(t)|}+\widetilde{A} .
\end{gathered}
$$

Then $h(t)=\chi_{|g(t)|}+\widetilde{A}$ is not integrable in the sense of Kaleva. However, the above system has the following solution:

$$
x(t)=\chi_{|G(t)|}+\widetilde{A} t,
$$

where

$$
G(t)= \begin{cases}t^{2} \sin \frac{1}{t^{2}}, & t \neq 0, \\ 0, & t=0 .\end{cases}
$$

It is well known that the Henstock integral is designed to integrate highly oscillatory functions which the Lebesgue integral fails to do. It is known as nonabsolute integral and it is a powerful tool. It is well known that the Henstock integral includes the Riemann, improper Riemann, Lebesgue, and 
Newton integrals. Though such an integral was defined by Denjoy in 1912 and also by Perron in 1914, it was difficult to handle using their definitions. But with the Riemann-type definition introduced more recently by Henstock in 1963 and also independently by Kurzweil, the definition is now simple and furthermore the proof involving the integral also turns out to be easy. For more detailed results about the Henstock integral, we refer to [16]. Recently, Wu and Gong [17, 18] have combined the fuzzy set theory and nonabsolute integral theory and discussed the fuzzy Henstock integrals of fuzzynumber-valued functions which extended Kaleva [1] integration. In order to complete the theory of fuzzy calculus and to transfer a fuzzy differential equation into a fuzzy integral equation, we $[19,20]$ have defined the strong fuzzy Henstock integrals and discussed some of their properties and the controlled convergence theorem.

In this paper, according to the idea of $[6,21,22]$ and using the concept of generalized differentiability [8], we will deal with the Cauchy problem of discontinuous fuzzy systems as follows:

$$
x(t)=\tilde{f}(t)+\int_{0}^{a} k_{1}(t, s) x(s) \mathrm{d} s+\int_{0}^{a} k_{2}(t, s) \tilde{g}(s, x(s)) \mathrm{d} s,
$$

where $t \in I_{a}=[0, a], a \in R^{+}$, and $x, \tilde{f}, \tilde{g}: I_{\alpha} \rightarrow E^{n}$ are fuzzy-number-valued function and integrals which are taken in sense of strong fuzzy Henstock integration, and $k_{1}, k_{2}: I_{a} \times$ $I_{a} \rightarrow R^{+}$are measurable functions such that $k_{1}(t, \cdot), k_{2}(t, \cdot)$ are continuous.

\section{Preliminaries}

2.1. Fuzzy Number Theory. Let $P_{k}\left(R^{n}\right)$ denote the family of all nonempty compact convex subset of $R^{n}$ and define the addition and scalar multiplication in $P_{k}\left(R^{n}\right)$ as usual. Let $A$ and $B$ be two nonempty bounded subsets of $R^{n}$. The distance between $A$ and $B$ is defined by the Hausdorff metric [10]

$$
d_{H}(A, B)=\max \left\{\sup _{a \in A} \inf _{b \in B}\|a-b\|, \sup _{b \in B} \inf _{a \in A}\|b-a\|\right\} .
$$

Denote $E^{n}=\left\{u: R^{n} \rightarrow[0,1] \mid u\right.$ satisfies (1)-(4) below $\}$, where

(1) $u$ is normal; that is, there exists an $x_{0} \in R^{n}$ such that $u\left(x_{0}\right)=1$,

(2) $u$ is fuzzy convex; that is, $u(\lambda x+(1-\lambda) y) \geq$ $\min \{u(x), u(y)\}$ for any $x, y \in R^{n}$ and $0 \leq \lambda \leq 1$,

(3) $u$ is upper semicontinuous,

(4) $[u]^{0}=\operatorname{cl}\left\{x \in R^{n} \mid u(x)>0\right\}$ is compact.

Then it is easy to see that $E^{n}$ is a fuzzy number space.

For $0<\alpha \leq 1$, denote $[u]^{\alpha}=\left\{x \in R^{n} \mid u(x) \geq \alpha\right\}$. Then from the above conditions (1)-(4), it follows that the $\alpha$-level set $[u]^{\alpha} \in P_{k}\left(R^{n}\right)$ for all $0 \leq \alpha<1$.
According to Zadeh's extension principle, we have addition and scalar multiplication in the fuzzy number space $E^{n}$ as follows [10]:

$$
[u+v]^{\alpha}=[u]^{\alpha}+[v]^{\alpha}, \quad[k u]^{\alpha}=k[u]^{\alpha},
$$

where $u, v \in E^{n}$ and $0 \leq \alpha \leq 1$.

Define $D: E^{n} \times E^{n} \rightarrow[0, \infty)$

$$
D(u, v)=\sup \left\{d_{H}\left([u]^{\alpha},[v]^{\alpha}\right): \alpha \in[0,1]\right\},
$$

where $d$ is the Hausdorff metric defined in $P_{k}\left(R^{n}\right)$. Then it is easy to see that $D$ is a metric in $E^{n}$. Using the results in [23], we know that

(1) $\left(E^{n}, D\right)$ is a complete metric space;

(2) $D(u+w, v+w)=D(u, v)$ for all $u, v, w \in E^{n}$;

(3) $D(\lambda u, \lambda v)=|\lambda| D(u, v)$ for all $u, v, w \in E^{n}$ and $\lambda \in R$.

The metric space $\left(E^{n}, D\right)$ has a linear structure; it can be embedded isomorphically as a cone in a Banach space of function $u^{*}: I \times S^{n-1} \rightarrow R$, where $S^{n-1}$ is the unit sphere in $R^{n}$, with an embedded function $u^{*}=j(u)$ defined by

$$
u^{*}(r, x)=\sup _{\alpha \in[u]^{\alpha}}\langle\alpha, x\rangle
$$

for all $\langle r, x\rangle \in I \times S^{n-1}$ (see [23]).

Theorem 2 (see [24]). There exists a real Banach space X such that $E^{n}$ can be embed as a convex cone $C$ with vertex 0 into $X$. Furthermore the following conditions hold true:

(1) the embedding $j$ is isometric;

(2) addition in $X$ induces addition in $E^{n}$;

(3) multiplication by nonnegative real number in $X$ induces the corresponding operation in $E^{n}$;

(4) $C-C$ is dense in $X$;

(5) $C$ is closed.

It is well known that the $H$-derivative for fuzzy-numberfunctions was initially introduced by Puri and Ralescu [5] and it is based on the condition $(H)$ of sets. We note that this definition is fairly strong, because the family of fuzzynumber-valued functions $H$-differentiable is very restrictive. For example, the fuzzy-number-valued function $\widetilde{f}:[a, b] \rightarrow$ $E^{n}$ defined by $\widetilde{f}(x)=C \cdot g(x)$, where $C$ is a fuzzy number, - is the scalar multiplication (in the fuzzy context), and $g$ : $[a, b] \rightarrow R^{+}$, with $g^{\prime}\left(t_{0}\right)<0$, is not $H$-differentiable in $t_{0}$ (see $[8,9]$ ). To avoid the above difficulty, in this paper we consider a more general definition of a derivative for fuzzynumber-valued functions enlarging the class of differentiable fuzzy-number-valued functions, which has been introduced in [8].

Definition 3 (see [8]). Let $\tilde{f}:(a, b) \rightarrow E^{n}$ and $x_{0} \in(a, b)$. We say that $\tilde{f}$ is differentiable at $x_{0}$, if there exists an element $\tilde{f}^{\prime}\left(t_{0}\right) \in E^{n}$, such that, 
(1) for all $h>0$ sufficiently small, there exists $\tilde{f}\left(x_{0}+\right.$ $h){ }_{H} \tilde{f}\left(x_{0}\right), \tilde{f}\left(x_{0}\right){ }_{H} \tilde{f}\left(x_{0}-h\right)$ and the limits (in the metric $D$ )

$$
\begin{aligned}
\lim _{h \rightarrow 0} & \frac{\tilde{f}\left(x_{0}+h\right)-_{H} \tilde{f}\left(x_{0}\right)}{h} \\
\quad & \lim _{h \rightarrow 0} \frac{\tilde{f}\left(x_{0}\right)-_{H} \tilde{f}\left(x_{0}-h\right)}{h}=\tilde{f}^{\prime}\left(x_{0}\right)
\end{aligned}
$$

or

(2) for all $h>0$ sufficiently small, there exists $\tilde{f}\left(x_{0}\right)$ ${ }_{-H} \tilde{f}\left(x_{0}+h\right), \tilde{f}\left(x_{0}-h\right)-_{H} \tilde{f}\left(x_{0}\right)$ and the limits

$$
\begin{aligned}
\lim _{h \rightarrow 0} & \frac{\tilde{f}\left(x_{0}\right)-_{H} \tilde{f}\left(x_{0}+h\right)}{-h} \\
\quad & \lim _{h \rightarrow 0} \frac{\tilde{f}\left(x_{0}-h\right)-{ }_{H} \tilde{f}\left(x_{0}\right)}{-h}=\tilde{f}^{\prime}\left(x_{0}\right)
\end{aligned}
$$

or

(3) for all $h>0$ sufficiently small, there exists $\tilde{f}\left(x_{0}+\right.$ $h){ }_{H} \tilde{f}\left(x_{0}\right), \tilde{f}\left(x_{0}-h\right){ }_{-} \tilde{f}\left(x_{0}\right)$ and the limits

$$
\begin{aligned}
& \lim _{h \rightarrow 0} \frac{\tilde{f}\left(x_{0}+h\right)-{ }_{H} \tilde{f}\left(x_{0}\right)}{h} \\
& \quad=\lim _{h \rightarrow 0} \frac{\tilde{f}\left(x_{0}-h\right)-{ }_{H} \tilde{f}\left(x_{0}\right)}{-h}=\tilde{f}^{\prime}\left(x_{0}\right)
\end{aligned}
$$

or

(4) for all $h>0$ sufficiently small, there exists $\tilde{f}\left(x_{0}\right)$ ${ }_{-}{ }_{H} \tilde{f}\left(x_{0}+h\right), \widetilde{f}\left(x_{0}\right){ }_{H} \tilde{f}\left(x_{0}-h\right)$ and the limits

$$
\begin{aligned}
\lim _{h \rightarrow 0} \frac{\tilde{f}\left(x_{0}\right)-{ }_{H} \tilde{f}\left(x_{0}+h\right)}{-h} \\
\quad=\lim _{h \rightarrow 0} \frac{\tilde{f}\left(x_{0}\right)-{ }_{H} \tilde{f}\left(x_{0}-h\right)}{h}=\tilde{f}^{\prime}\left(x_{0}\right)
\end{aligned}
$$

( $h$ and $-h$ at denominators mean $(1 / h)$. and $-(1 / h)$, resp.).

2.2. The Strong Henstock Integrals of Fuzzy-Number-Valued Functions in $E^{n}$. In this section we define the strong Henstock integrals of fuzzy-number-valued functions in the fuzzy number space $E^{n}$ and we give some properties of this integral.

Definition 4 (see [20]). A fuzzy-number-valued function $\tilde{f}$ will be termed piecewise additive on $[a, b]$ if there exists a division $T: a=a_{0}<a_{1}<\cdots<a_{n}=b$, such that $\widetilde{f}(x)$ is additive on each $\left[a_{i}, a_{i+1}\right](i=0,1, \ldots, n-1)$.

Definition 5 (see $[19,20])$. A fuzzy-number-valued function $\tilde{f}$ is said to be strong Henstock integrable on $[a, b]$ if there exists a piecewise additive fuzzy-number-valued function $\widetilde{F}$ on $[a, b]$ such that for every $\varepsilon>0$ there exists a function $\delta(\xi)>0$ and for any $\delta$-fine division $P=\left\{\left[x_{i-1}, x_{i}\right], \xi_{i}\right\}_{i=1}^{n}$ of $[a, b]$ we have

$$
\begin{aligned}
(P) \sum_{i \in K_{n}} D\left(\tilde{f}\left(\xi_{i}\right)\left(x_{i}-x_{i-1}\right), \widetilde{F}\left(\left[x_{i-1}, x_{i}\right]\right)\right) \\
+(P) \sum_{j \in I_{n}} D\left(\widetilde{f}\left(\xi_{j}\right)\left(x_{j}-x_{j-1}\right),\right. \\
\left.(-1) \cdot \widetilde{F}\left(\left[x_{j}, x_{j-1}\right]\right)\right)<\varepsilon,
\end{aligned}
$$

where $K_{n}=\left\{i \in\{1,2, \ldots, n\}\right.$ such that $\widetilde{F}\left(\left[x_{i-1}, x_{i}\right]\right)$ is a fuzzy number and $I_{n}=\left\{j \in\{1,2, \ldots, n\}\right.$ such that $\widetilde{F}\left(\left[x_{j}, x_{j-1}\right]\right)$ is a fuzzy number. We write $\widetilde{f} \in S F H[a, b]$.

Definition 6 (see [20]). A fuzzy-number-valued function $\widetilde{F}$ defined on $X \subset[a, b]$ is said to be $A C^{*}(X)$ if for every $\varepsilon>0$ there exists $\eta>0$ such that for every finite sequence of nonoverlapping intervals $\left\{\left[a_{i}, b_{i}\right]\right\}$, satisfying $\sum_{i=1}^{n}\left|b_{i}-a_{i}\right|<\eta$ where $a_{i}, b_{i} \in X$ for all $i$, we have

$$
\sum \omega\left(\widetilde{F},\left[a_{i}, b_{i}\right]\right)<\varepsilon,
$$

where $\omega$ denotes the oscillation of $\widetilde{F}$ over $\left[a_{i}, b_{i}\right]$; that is,

$$
\omega\left(\widetilde{F},\left[a_{i}, b_{i}\right]\right)=\sup \left\{D(\widetilde{F}(y), \widetilde{F}(x)) ; x, y \in\left[a_{i}, b_{i}\right]\right\} .
$$

Definition 7 (see [20]). A fuzzy-number-valued function $\widetilde{F}$ is said to be $A C G^{*}$ on $X$ if $X$ is the union of a sequence of closed sets $\left\{X_{i}\right\}$ such that, on each $X_{i}, \widetilde{F}$ is $A C^{*}\left(X_{i}\right)$.

For the strong fuzzy Henstock integrable we have the following theorems.

Theorem 8. Let $\tilde{f}:[a, b] \rightarrow E^{n}$. If $\tilde{f}=0$ a.e on $[a, b]$, then $\tilde{f}$ is SFH integrable on $[a, b]$ and $\int_{a}^{b} \widetilde{f}(t) \mathrm{d} t=0$.

Theorem 9. Let $\tilde{f}:[a, b] \rightarrow E^{n}$ be SFH integrable on $[a, b]$ and let $\widetilde{F}(x)=\int_{a}^{x} \tilde{f}(t) \mathrm{d} t$ for each $x \in[a, b]$. Then

(a) the function $\widetilde{F}$ is continuous on $[a, b]$;

(b) the function $\widetilde{F}$ is differentiable a.e on $[a, b]$ and $\widetilde{F}^{\prime}=f$;

(c) $\tilde{f}$ is measurable.

Theorem 10 (controlled convergence theorem; see [20]). Suppose that $\left\{\widetilde{f}_{n}\right\}$ is a sequence of SFH integrable functions on $[a, b]$ satisfying the following conditions:

(1) $\tilde{f}_{n}(x) \rightarrow \widetilde{f}(x)$ a.e. in $[a, b]$ as $n \rightarrow \infty$;

(2) the primitives $\widetilde{F}_{n}$ of $\widetilde{f}_{n}$ are $A C G^{*}$ uniformly in $n$;

(3) the primitives $\widetilde{F}_{n}$ converge uniformly on $[a, b]$;

then $\tilde{f}$ is also SFH integrable on $[a, b]$ and

$$
\lim _{n \rightarrow \infty} \int_{a}^{b} \tilde{f}_{n}(x) \mathrm{d} x=\int_{a}^{b} \tilde{f}(x) \mathrm{d} x .
$$




\section{Main Results}

In this section we prove some existence theorems for the problem (4).

For any bounded subset $A$ of the Banach space $X$, we denote by $\alpha(A)$ the Kuratowski measure of noncompactness of $A$; that is, the infimum of all $\varepsilon>0$ such that there exists a finite covering of $A$ by sets of diameter less than $\varepsilon$. For the properties of $\alpha$ we refer to [25], for example.

Lemma 11 (see [25]). Let $H \subset C\left(I_{\gamma}, X\right)$ be a family of strong equicontinuous functions; then

$$
\alpha_{c}(H)=\sup _{t \in I_{\gamma}} \alpha(H(t))=\alpha\left(H\left(I_{\gamma}\right)\right),
$$

where $\alpha_{c}(H)$ denotes the Kuratowski measure of noncompactness in $C\left(I_{\gamma}, X\right)$ and the function $t \rightarrow \alpha(H(t))$ is continuous.

Theorem 12 (see [25]). Let $D$ be a closed convex subset of $X$ and let $F$ be a continuous function from $D$ into itself. If, for $x \in D$,

$$
\bar{V}=\overline{\operatorname{con}}(\{x\} \cup F(V)) \Longrightarrow V
$$

is relatively compact, then $F$ has a fixed point.

Theorem 13. If the fuzzy-number-valued function $\tilde{f}: I_{a} \rightarrow$ $E^{n}$ is (SFH) integrable, then

$$
\int_{I} \tilde{f}(t) \mathrm{d} t \in|I| \cdot \overline{\operatorname{conv}} \widetilde{f}(I),
$$

where $\overline{\operatorname{conv}} \tilde{f}(I)$ is the convex hull of $\tilde{f}(I), I$ is an arbitrary subinterval of $I_{a}$, and $|I|$ is the length of $I$.

Proof. Because $j \circ \tilde{f}$ is abstract $(H)$ integrable in a Banach Space, by using the mean valued theorem of $(H)$ integrals, we have

$$
(H) \int_{I} j \circ \tilde{f}(t) \mathrm{d} t \in|I| \cdot \overline{\operatorname{conv}} j \circ \tilde{f}(I)=|I| \cdot j \circ \overline{\operatorname{conv}} \tilde{f}(t) .
$$

On the other hand, there exists $(H) \int_{I} j \circ \tilde{f}(t) \mathrm{d} t=j \circ \int_{I} \tilde{f}(t) \mathrm{d} t$.

So, we have $j \circ \int_{I} \widetilde{f}(t) \mathrm{d} t \in|I| \cdot \overline{\operatorname{conv}} j \circ \widetilde{f}(I)$. And the set $\{|I| \cdot \overline{\operatorname{conv}} \tilde{f}(I)\}$ is a closed convex set; we have

$$
\int_{I} \tilde{f}(t) \mathrm{d} t \in|I| \cdot \overline{\operatorname{conv}} \tilde{f}(I) .
$$

Definition 14. A fuzzy valued function $\tilde{f}: I_{\alpha} \times E^{n} \rightarrow E^{n}$ is a Caratheodory function if, for each $x \in E^{n}$, the fuzzy valued function $\tilde{f}(t, x)$ is measurable in $t \in I_{\alpha}$, and for almost all $t \in I_{\alpha}$, the fuzzy valued function $\widetilde{f}(t, x)$ is continuous with respect to $x$.

For $x \in C\left(I_{a}, E^{n}\right)$, we define the norm of $x$ by

$$
H(x, \widetilde{0})=\sup _{t \in I_{a}} D(x, \widetilde{0}) .
$$

Let

$$
\begin{aligned}
& B(p)=\left\{x \in C\left(I_{a}, E^{n}\right) \mid\right. \\
&H(x, \widetilde{0}) \leq H(\widetilde{f}(\cdot), \widetilde{0})+p, p>0\} .
\end{aligned}
$$

Obviously, the set $B(p)$ is closed and convex in $E^{n}$.

We define the operator $F: C\left(I_{a}, E^{n}\right) \rightarrow C\left(I_{a}, E^{n}\right)$ by

$$
\begin{aligned}
F(x)(t)= & \tilde{f}(t)+\int_{0}^{a} k_{1}(t, s) x(s) \mathrm{d} s \\
& +\int_{0}^{a} k_{2}(t, s) \tilde{g}(s, x(s)) \mathrm{d} s, \quad t \in I_{a}, x \in B(p),
\end{aligned}
$$

where integrals are taken in the sense of SFH. Moreover, let $\Gamma(p)=\left\{F(x) \in C\left(I_{a}, E^{n}\right) \mid x \in B(p)\right\}$.

Definition 15. A continuous function $x: I_{a} \rightarrow E^{n}$ is said to be a solution of the problem (4), if $x(t)$ satisfies

$$
x(t)=\tilde{f}(t)+\int_{0}^{a} k_{1}(t, s) x(s) \mathrm{d} s+\int_{0}^{a} k_{2}(t, s) \tilde{g}(s, x(s)) \mathrm{d} s
$$

or

$$
\begin{aligned}
x(t)= & \tilde{f}(t)+(-1) \cdot \int_{0}^{a} k_{1}(t, s) x(s) \mathrm{d} s \\
& +(-1) \cdot \int_{0}^{a} k_{2}(t, s) \tilde{g}(s, x(s)) \mathrm{d} s, \quad t \in I_{a} .
\end{aligned}
$$

Theorem 16. Assume that, for each continuous function $x(t)$, $\tilde{g}(\cdot, x(\cdot))$ is $(S F H)$ integrable, and $\widetilde{g}$ is a Caratheodory function. Let $k_{1}, k_{2}: I_{a} \times I_{a} \rightarrow R^{+}$be measure functions such that $k_{1}(t, \cdot), k_{2}(t, \cdot)$ are continuous. Moreover, there exists $P_{0}>0$ and a Caratheodory function $\omega: I_{a} \times R^{+} \rightarrow R^{+}$, with

$$
\begin{gathered}
\alpha(j \circ \tilde{g}(s, X)) \leq \omega(s, \alpha(j \circ X)), \\
\text { a.e. } s \in I_{a}, \quad X \subset B\left(p_{0}\right),
\end{gathered}
$$

such that the zero function is the unique continuous solution of the inequality

$$
q(t) \leq 2\left[\int_{0}^{c} k_{1}(t, s) q(t, s) \mathrm{d} s+\int_{0}^{c} k_{2}(t, s) \omega(s, q(s)) \mathrm{d} s\right] .
$$

Suppose that $\Gamma\left(p_{0}\right)$ is equicontinuous, equibounded, and uniformly $A C G^{*}$ on $I_{a}$. Then there exists at least a solution of the problem (4) on $I_{a}$ for some $0<c \leq a$ with continuous initial function $\widetilde{f}$. 
Proof. By equicontinuity and equiboundedness of $\Gamma\left(p_{0}\right)$, there exist some numbers $c(0<c \leq a)$ such that

$$
\begin{gathered}
H\left(\int_{0}^{c}\left[k_{1}(t, s) x(s)+k_{2}(t, s) \tilde{g}(s, x(s))\right] \mathrm{d} s, \tilde{0}\right) \\
=\sup _{t \in I_{c}} D\left(\int_{0}^{c}\left[k_{1}(t, s) x(s)+k_{2}(t, s) \tilde{g}(s, x(s))\right] \mathrm{d} s, \tilde{0}\right) \\
=\sup _{t \in I_{c}} \max _{r \in[0,1]}\left\{\mid \int_{0}^{c}\left[k_{1}(t, s) x_{r}^{+}(s)\right.\right. \\
\left.\quad+k_{2}(t, s) \tilde{g}_{r}^{+}(s, x(s))\right] \mathrm{d} s-\widetilde{0}_{r}^{+} \mid, \\
\mid \int_{0}^{c}\left[k_{1}(t, s) x_{r}^{-}(s)\right. \\
\left.\left.+k_{2}(t, s) \tilde{g}_{r}^{-}(s, x(s))\right] \mathrm{d} s-\tilde{0}_{r}^{-} \mid\right\} \leq p_{0}
\end{gathered}
$$

for $t \in I_{c}$ and $x \in B\left(p_{0}\right)$.

Next, we will prove that the operator $F$ is continuous. In fact, let $x_{n} \rightarrow x$. Because the function $\widetilde{g}$ is a Caratheodory function, by the following equality

$$
\begin{aligned}
& H\left(F\left(x_{n}\right), F(x)\right) \\
& =H\left(\int _ { 0 } ^ { c } \left(k_{1}(t, s)\left(x_{n}(s)-x(s)\right)+k_{2}(t, s)\right.\right. \\
& \left.\left.\quad \times\left(\tilde{g}\left(s, x_{n}(s)\right)-\tilde{g}(s, x(s))\right)\right) \mathrm{d} s, \tilde{0}\right) \\
& =\sup _{t \in I_{c}} D\left(\int _ { 0 } ^ { c } \left(k_{1}(t, s)\left(x_{n}(s)-x(s)\right)+k_{2}(t, s)\right.\right. \\
& \left.\left.\quad \times\left(\tilde{g}\left(s, x_{n}(s)\right)-\tilde{g}(s, x(s))\right)\right) \mathrm{d} s, \tilde{0}\right)
\end{aligned}
$$

and Theorem 10, we have $F\left(x_{n}\right) \rightarrow F(x)$.

Observe that a fixed point of $F$ is the solution of the problem (4). Now we prove that $F$ has a fixed point using Theorem 12.

Suppose that $V(t)=\left\{v(t) \in E^{n} \mid v \in V\right\} \subset B\left(p_{0}\right)$ satisfies condition $\bar{V}=\overline{\operatorname{conv}}(\{x\} \cup F(V))$ for some $x \in B\left(p_{0}\right), t \in I_{c}$. Let $V \subset B\left(p_{0}\right), F(V) \subset \Delta\left(p_{0}\right)$; then $V \subset \bar{V}$ is equicontinuous. By Lemma 11, $t \rightarrow v(t)=\alpha(j \circ V(t))$ is continuous on $I_{c}$.

Let $\int_{0}^{c} Z(s) \mathrm{d} s=\left\{\int_{0}^{c} x(s) \mathrm{d} s \mid x \in Z\right\}$ for any $Z \in C\left(I_{c}, E^{n}\right)$ and let $\widetilde{h}$ denote the mapping defined by $\widetilde{h}(x(s))=\widetilde{g}(s, x(s))$, for each $x \in B\left(p_{0}\right), s \in I_{c}$. Obviously, $\widetilde{h}(V(s))=\widetilde{g}(s, V(s))$, and

$$
F(V(t))=\tilde{f}(t)+\int_{0}^{c}\left[k_{1}(t, s) V(s)+k_{2}(t, s) \tilde{h}(V(s))\right] \mathrm{d} s
$$

holds ture.
Using (27), Lemma 11, and the properties of measure of noncompactness $\alpha$, we have

$$
\begin{aligned}
& \alpha(j \circ F(V(t))) \\
& =\alpha\left(j \circ \left(\tilde{f}(t)+\int_{0}^{c}\left[k_{1}(t, s) V(s)\right.\right.\right. \\
& \left.\left.\left.+k_{2}(t, s) \tilde{h}(V(s))\right] \mathrm{d} s\right)\right) \\
& \leq 2 \alpha\left(j \circ\left(\int_{0}^{c}\left[k_{1}(t, s) V(s)+k_{2}(t, s) \tilde{h}(V(s))\right] \mathrm{d} s\right)\right) \\
& \leq 2 \int_{0}^{c}\left[k_{1}(t, s) \alpha(j \circ V(s))\right. \\
& \left.\quad+k_{2}(t, s) \alpha(j \circ \tilde{g}(s, V(s)))\right] \mathrm{d} s \\
& \leq 2 \int_{0}^{c}\left[k_{1}(t, s) \alpha(j \circ V(s))\right. \\
& \left.+k_{2}(t, s) \omega(s, \alpha(j \circ V(s)))\right] \mathrm{d} s .
\end{aligned}
$$

Because $V=\overline{\operatorname{conv}}(\{x\} \cup F(V))$, we have

$$
v(t) \leq 2\left[\int_{0}^{c} k_{1}(t, s) v(s) \mathrm{d} s+\int_{0}^{c} k_{2}(t, s) \omega(s, v(s)) \mathrm{d} s\right] .
$$

By assumption, because the zero function is unique continuous solution of the last inequality, so we have $v(t)=\alpha(j$ 。 $V(t))=0$. By Arzelá-Ascoli Theorem, $V$ is relatively compact. So, by Theorem $12, F$ has a fixed point which is a solution of problem (4).

Next, we give another existence theorem for problem (4).

Let $\gamma(K)$ be the spectral radius of the integral operator $K$ defined by

$$
\begin{array}{r}
K(u)(t)=\int_{0}^{a}\left(k_{1}(t, s)+k_{2}(t, s)\right) u(s) \mathrm{d} s, \\
u \in B\left(p_{0}\right), \quad t \in I_{\alpha} .
\end{array}
$$

Theorem 17. Assume that, for each continuous function $x(t)$, $\tilde{g}(\cdot, x(\cdot))$ is (SFH) integrable, and $\widetilde{g}$ is a Caratheodory function and $k_{1}, k_{2}: I_{a} \times I_{a} \rightarrow R^{+}$are measure functions such that $k_{1}(t, \cdot), k_{2}(t, \cdot)$ are continuous. Moreover, there exists $P_{0}>0$ and $L>0$ such that

$$
\alpha(j \circ \tilde{g}(I, X)) \leq L \alpha(j \circ X)
$$

for each $I \subset I_{a}, X \subset B\left(p_{0}\right)$. Suppose that $\Gamma\left(p_{0}\right)$ is equicontinuous, equibounded, and uniformly $A C G^{*}$ on $I_{a}$ and $(1+$ $L) \gamma(K)<1$. Then there exists at least a solution of the problem (4) on $I_{a}$ for some $0<c \leq a$ with continuous initial function $\tilde{f}$.

Proof. By equicontinuity and equiboundedness of $\Gamma\left(p_{0}\right)$, there exist some numbers $c(0<c \leq a)$ such that

$$
H\left(\int_{0}^{c}\left(k_{1}(t, s)+k_{2}(t, s)\right) u(s) \mathrm{d} s, \tilde{0}\right) \leq p_{0}
$$


for $t \in p_{0}$ and $x \in B\left(p_{0}\right)$. By assumption, the operator $F$ is well defined and maps $B\left(p_{0}\right)$ into $B\left(p_{0}\right)$. Now, we show that the operator $F$ is continuous. In fact, let $x_{n} \rightarrow x$. Because the function $\widetilde{g}$ is a Caratheodory function, by following equality

$$
\begin{aligned}
& H\left(F\left(x_{n}\right), F(x)\right) \\
& =H\left(\int _ { 0 } ^ { c } \left(k_{1}(t, s)\left(x_{n}(s)-x(s)\right)+k_{2}(t, s)\right.\right. \\
& \left.\left.\quad \times\left(\tilde{g}\left(s, x_{n}(s)\right)-\tilde{g}(s, x(s))\right)\right) \mathrm{d} s, \tilde{0}\right) \\
& =\sup _{t \in I_{c}} D\left(\int _ { 0 } ^ { c } \left(k_{1}(t, s)\left(x_{n}(s)-x(s)\right)+k_{2}(t, s)\right.\right. \\
& \left.\left.\quad \times\left(\tilde{g}\left(s, x_{n}(s)\right)-\tilde{g}(s, x(s))\right)\right) \mathrm{d} s, \tilde{0}\right)
\end{aligned}
$$

and Theorem 10, we have $F\left(x_{n}\right) \rightarrow F(x)$.

Observe that a fixed point of $F$ is the solution of the problem (4). Now we prove that $F$ has a fixed point using Theorem 12.

Suppose that $V(t)=\left\{v(t) \in E^{n} \mid v \in V\right\} \subset B\left(p_{0}\right)$ satisfies condition $\bar{V}=\overline{\operatorname{conv}}(\{x\} \cup F(V))$ for some $x \in B\left(p_{0}\right), t \in I_{c}$. Let $V \subset B\left(p_{0}\right), F(V) \subset \Delta\left(p_{0}\right)$; then $V \subset \bar{V}$ is equicontinuous. By Lemma 11, $t \rightarrow v(t)=\alpha(j \circ V(t))$ is continuous on $I_{c}$.

We divide the interval $I_{c}: 0=t_{0}<t_{1}<t_{2}<\cdots<t_{m}=c$, where $t_{i}=i c / m, i=0,1, \ldots, m$. Let $V\left(\left[t_{i}, t_{i+1}\right]\right)=\{u(s) \in$ $\left.E^{n}: u \in V, t_{i} \leq s \leq t_{t+1}, i=0,1, \ldots, m-1\right\}$. By Lemma 11 and the continuity of $v$ there exists $s_{i} \in T_{i}=\left[t_{i}, t_{i+1}\right]$ such that

$$
\begin{aligned}
\alpha\left(j \circ V\left(\left[t_{i}, t_{i+1}\right]\right)\right) & =\sup \left\{\alpha(j \circ V(s)): t_{i} \leq s \leq t_{t+1}\right\} \\
& =v\left(s_{i}\right) .
\end{aligned}
$$

In addition, by the definition of operator $F$ and Theorem 16 we have

$F(u)(t)$

$$
\begin{gathered}
=\tilde{f}(t)+\sum_{i=0}^{m-1} \int_{t_{i}}^{t_{i}+1}\left[k_{1}(t, s) u(s)\right. \\
\left.\quad+k_{2}(t, s) \tilde{g}(s, u(s))\right] \mathrm{d} s \\
\in \tilde{f}(t)+\sum_{i=0}^{m-1}\left(t_{i-1}-t_{i}\right) \\
\quad \times \frac{\operatorname{conv}}{\left[k_{1}\left(t, T_{i}\right) V\left(T_{i}\right)\right.} \\
\left.\quad+k_{2}\left(t, T_{i}\right) \tilde{g}\left(T_{i}, V\left(T_{i}\right)\right)\right]
\end{gathered}
$$

for all $u \in V$, where $k_{m}\left(t, T_{i}\right)=\left\{k_{m}(t, s), t, s \in T_{i}\right\}$ and $\tilde{g}\left(T_{i}\right.$, $\left.V\left(T_{i}\right)\right)=\left\{\widetilde{g}(t, x(t)): t \in T_{i}, x \in V\right\}$. So, we have

$F(V)(t)$

$$
\begin{aligned}
\subset \tilde{f}(t)+\sum_{i=0}^{m-1}\left(t_{i-1}-t_{i}\right) \overline{\operatorname{conv}}[ & k_{1}\left(t, T_{i}\right) V\left(T_{i}\right) \\
& \left.+k_{2}\left(t, T_{i}\right) \tilde{g}\left(T_{i}, V\left(T_{i}\right)\right)\right] .
\end{aligned}
$$

Using (35), (38) and the properties of measure of noncompactness $\alpha$, we have

$$
\begin{aligned}
\alpha & (j \circ F(V)(t)) \\
\leq & \sum_{i=0}^{m-1}\left(t_{i+1}-t_{i}\right)\left[k_{1}\left(t, T_{i}\right) j \circ v\left(s_{i}\right)+k_{2}\left(t, T_{i}\right) L \cdot j \circ v\left(s_{i}\right)\right] \\
= & \sum_{i=0}^{m-1}\left(t_{i+1}-t_{i}\right) k_{1}\left(t, T_{i}\right) j \circ v\left(s_{i}\right) \\
& +L \sum_{i=0}^{m-1}\left(t_{i+1}-t_{i}\right) k_{2}\left(t, T_{i}\right) j \circ v\left(s_{i}\right) \\
\leq & \sum_{i=0}^{m-1}\left(t_{i+1}-t_{i}\right) \sup _{s_{i} \in T_{i}} k_{1}\left(t, s_{i}\right) j \circ v\left(s_{i}\right) \\
& +L \cdot \sum_{i=0}^{m-1}\left(t_{i+1}-t_{i}\right) \sup _{s_{i} \in T_{i}} k_{2}\left(t, s_{i}\right) j \circ v\left(s_{i}\right) \\
& \sum_{i=0}^{m-1}\left(t_{i+1}-t_{i}\right) k_{1}\left(t, p_{i}\right) j \circ v\left(s_{i}\right) \\
& +L \cdot \sum_{i=0}^{m-1}\left(t_{i+1}-t_{i}\right) k_{2}\left(t, q_{i}\right) j \circ v\left(s_{i}\right),
\end{aligned}
$$

where $s_{i}, p_{i}, q_{i} \in T_{i}$; so we get

$$
\begin{aligned}
\alpha(j \circ & F(V)(t)) \\
\leq & \sum_{i=0}^{m-1}\left(t_{i+1}-t_{i}\right) k_{1}\left(t, p_{i}\right) j \circ v\left(p_{i}\right) \\
& +\sum_{i=0}^{m-1}\left(t_{i+1}-t_{i}\right)\left[k_{1}\left(t, p_{i}\right) j \circ\left(v\left(s_{i}\right)-v\left(p_{i}\right)\right)\right] \\
& +L \sum_{i=0}^{m-1}\left(t_{i+1}-t_{i}\right) k_{2}\left(t, q_{i}\right) j \circ v\left(q_{i}\right) \\
& +L \cdot \sum_{i=0}^{m-1}\left(t_{i+1}-t_{i}\right)\left[k_{2}\left(t, q_{i}\right) j \circ\left(v\left(s_{i}\right)-v\left(q_{i}\right)\right)\right] \\
= & \sum_{i=0}^{m-1}\left(t_{i+1}-t_{i}\right) k_{1}\left(t, p_{i}\right) j \circ v\left(p_{i}\right) \\
& +\frac{c}{m} \sum_{i=0}^{m-1}\left[k_{1}\left(t, p_{i}\right) j \circ\left(v\left(s_{i}\right)-v\left(p_{i}\right)\right)\right] \\
& +\frac{L \cdot c}{m} \sum_{i=0}^{m-1}\left[k_{2}\left(t, q_{i}\right) j \circ\left(v\left(s_{i}\right)-v\left(q_{i}\right)\right)\right]
\end{aligned}
$$


By continuity of $v$ we have $v\left(s_{i}\right) \rightarrow v\left(p_{i}\right)<\varepsilon_{1}$ and $v\left(s_{i}\right) \rightarrow v\left(q_{i}\right)<\varepsilon_{2}$ as $m \rightarrow \infty$. So, we have

$$
\begin{aligned}
& \alpha(j \circ F(V)(t)) \\
& \quad<\int_{0}^{c} k_{1}(t, s) v(s) \mathrm{d} s+c \cdot \sup _{p \in I_{c}} k_{1}(t, p) \varepsilon_{1} \\
& \quad+L \cdot \int_{0}^{c} k_{2}(t, s) j \circ v(s) \mathrm{d} s+L \cdot c \cdot \sup _{q \in I_{c}} k_{2}(t, q) \varepsilon_{2} .
\end{aligned}
$$

Therefore, we have

$$
\begin{aligned}
\alpha(j \circ F(V)(t)) \leq & (1+L) \\
& \cdot j \circ \int_{0}^{c}\left[k_{1}(t, s)+k_{2}(t, s)\right] v(s) \mathrm{d} s
\end{aligned}
$$

for $t \in I_{c}$. Since $V=\overline{\operatorname{conv}}(\{u\} \cup F(V))$, by the properties of measure of noncompactness $\alpha$, we have

$$
\alpha(j \circ V(t)) \leq \alpha(j \circ(F(V)(t))),
$$

and so in view of (44) it follows that

$$
v(t) \leq(1+L) \int_{0}^{c}\left[k_{1}(t, s)+k_{2}(t, s)\right] v(s) \mathrm{d} s
$$

for $t \in I_{c}$. Because this inequality holds for all $t \in I_{c}$ and $(1+L) \gamma(K)<1$, by applying Gronwall's inequality, we get that $\alpha(j \circ V(t))=0$ for $t \in I_{c}$. By Arzelá-Ascoli Theorem, $V$ is relatively compact. So, by Theorem $12, F$ has a fixed point which is a solution of problem (4).

\section{Conclusion}

In this paper, we deal with the existence problems of discontinuous fuzzy integral equations involving the strong fuzzy Henstock integral in fuzzy number space. The functions of the equations are supposed to be discontinuous with respect to some variables and satisfy nonabsolute fuzzy integrability. Our result improves the result given in $[15,26]$ (where uniform continuity was required), as well as those referred to therein.

\section{Conflict of Interests}

The authors declare that there is no conflict of interests regarding the publication of this paper.

\section{Acknowledgments}

The authors are very grateful to the anonymous referees and Professor Márcia Federson for many valuable comments and suggestions which helped to improve the presentation of the paper. The authors would like to thank the National Natural Science Foundation of China (nos. 11161041 and 71061013) and the Fundamental Research Fund for the Central Universities (no. 31920130010).

\section{References}

[1] O. Kaleva, "Fuzzy differential equations," Fuzzy Sets and Systems, vol. 24, no. 3, pp. 301-319, 1987.

[2] Z. Gong and Y. Shao, "Global existence and uniqueness of solutions for fuzzy differential equations under dissipative-type conditions," Computers \& Mathematics with Applications, vol. 56, no. 10, pp. 2716-2723, 2008.

[3] O. Kaleva, "The Cauchy problem for fuzzy differential equations," Fuzzy Sets and Systems, vol. 35, no. 3, pp. 389-396, 1990.

[4] J. J. Nieto, "The Cauchy problem for continuous fuzzy differential equations," Fuzzy Sets and Systems, vol. 102, no. 2, pp. 259262, 1999.

[5] M. L. Puri and D. A. Ralescu, "Differentials of fuzzy functions," Journal of Mathematical Analysis and Applications, vol. 91, no. 2, pp. 552-558, 1983.

[6] C. Wu and S. Song, "Existence theorem to the Cauchy problem of fuzzy differential equations under compactness-type conditions," Information Sciences, vol. 108, no. 1-4, pp. 123-134, 1998.

[7] S. Seikkala, "On the fuzzy initial value problem," Fuzzy Sets and Systems, vol. 24, no. 3, pp. 319-330, 1987.

[8] B. Bede and S. G. Gal, "Generalizations of the differentiability of fuzzy-number-valued functions with applications to fuzzy differential equations," Fuzzy Sets and Systems, vol. 151, no. 3, pp. 581-599, 2005.

[9] Y. Chalco-Cano and H. Román-Flores, "On new solutions of fuzzy differential equations," Chaos, Solitons and Fractals, vol. 38, no. 1, pp. 112-119, 2008.

[10] D. Dubois and H. Prade, "Towards fuzzy differential calculus. I: integration of fuzzy mappings," Fuzzy Sets and Systems, vol. 8, no. 1, pp. 1-17, 1982.

[11] T. Allahviranloo, M. Khezerloo, O. Sedaghatfar, and S. Salahshour, "Toward the existence and uniqueness of solutions of second-order fuzzy volterra integro-differential equations with fuzzy kernel," Neural Computing and Applications, vol. 22, no. 1, supplement, pp. 133-141, 2013.

[12] J. Y. Park, Y. C. Kwun, and J. U. Jeong, "Existence of solutions of fuzzy integral equations in Banach spaces," Fuzzy Sets and Systems, vol. 72, no. 3, pp. 373-378, 1995.

[13] J. Y. Park and J. U. Jeong, "On the existence and uniqueness of solutions of fuzzy Volterra-Fredholm integral equations," Fuzzy Sets and Systems, vol. 115, no. 3, pp. 425-431, 2000.

[14] D. Zhang, W. Feng, and J. Qiu, "Global existence of solutions to fuzzy volterra integral equations," ICIC Express Letters, vol. 3, no. 3, pp. 707-711, 2009.

[15] X. Xue and Y. Fu, "Carathéodory solutions of fuzzy differential equations," Fuzzy Sets and Systems, vol. 125, no. 2, pp. 239-243, 2002.

[16] P. Y. Lee, Lanzhou Lectures on Henstock Integration, World Scientific, Singapore, 1989.

[17] C. Wu and Z. Gong, "On Henstock integrals of interval-valued functions and fuzzy-valued functions," Fuzzy Sets and Systems, vol. 115, no. 3, pp. 377-391, 2000

[18] C. Wu and Z. Gong, "On Henstock integral of fuzzy-numbervalued functions. I," Fuzzy Sets and Systems, vol. 120, no. 3, pp. 523-532, 2001.

[19] Z. Gong, "On the problem of characterizing derivatives for the fuzzy-valued functions. II. Almost everywhere differentiability and strong Henstock integral," Fuzzy Sets and Systems, vol. 145, no. 3, pp. 381-393, 2004. 
[20] Z. Gong and Y. Shao, "The controlled convergence theorems for the strong Henstock integrals of fuzzy-number-valued functions," Fuzzy Sets and Systems, vol. 160, no. 11, pp. 15281546, 2009.

[21] T. S. Chew and F. Flordeliza, "On $x^{\prime}=f(t, x)$ and HenstockKurzweil integrals," Differential and Integral Equations, vol. 4, no. 4, pp. 861-868, 1991.

[22] A. Sikorska-Nowak, "Existence of solutions of nonlinear integral equations and Henstock-Kurzweil integrals," Annales Societatis Mathematicae Polonae, vol. 47, no. 2, pp. 227-238, 2007.

[23] P. Diamond and P. Kloeden, Metric Space of Fuzzy Fets: Theory and Applications, World Scientific, Singapore, 1994.

[24] C. X. Wu and M. Ma, "Embedding problem of fuzzy number space. II," Fuzzy Sets and Systems, vol. 45, no. 2, pp. 189-202, 1992.

[25] J. Banaś and K. Goebel, Measure of Noncompactness in Banach Space, vol. 60 of Lecture Notes in Pure and Applied Mathematics, Marcel Dekker, New York, NY, USA, 1980.

[26] V. Lakshmikantham and R. N. Mohapatra, Theory of Fuzzy Differential Equations and Inclusions, Taylor \& Francis, London, UK, 2003. 


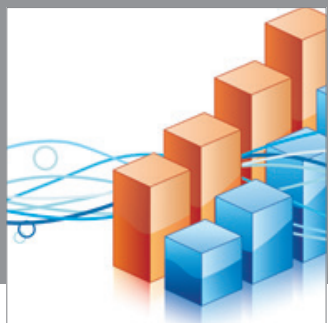

Advances in

Operations Research

mansans

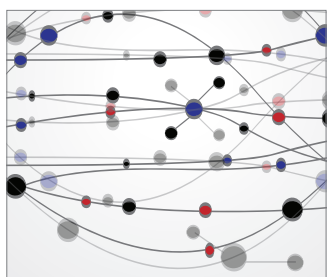

The Scientific World Journal
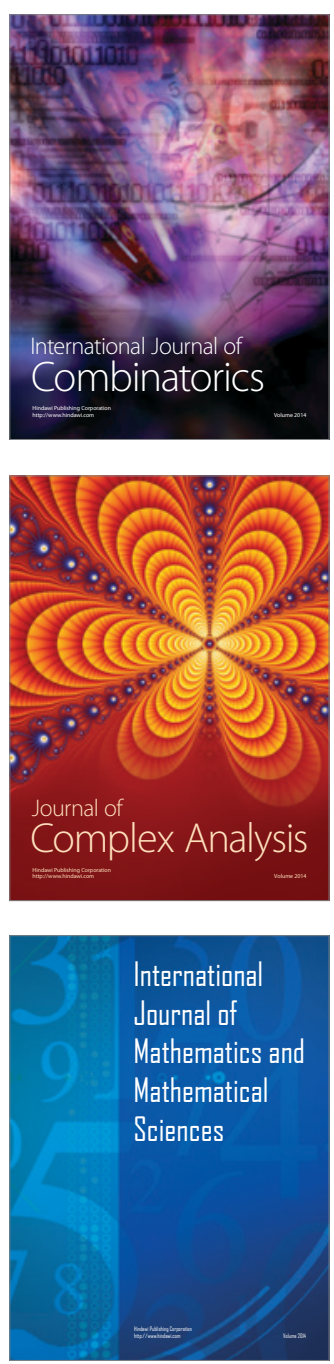
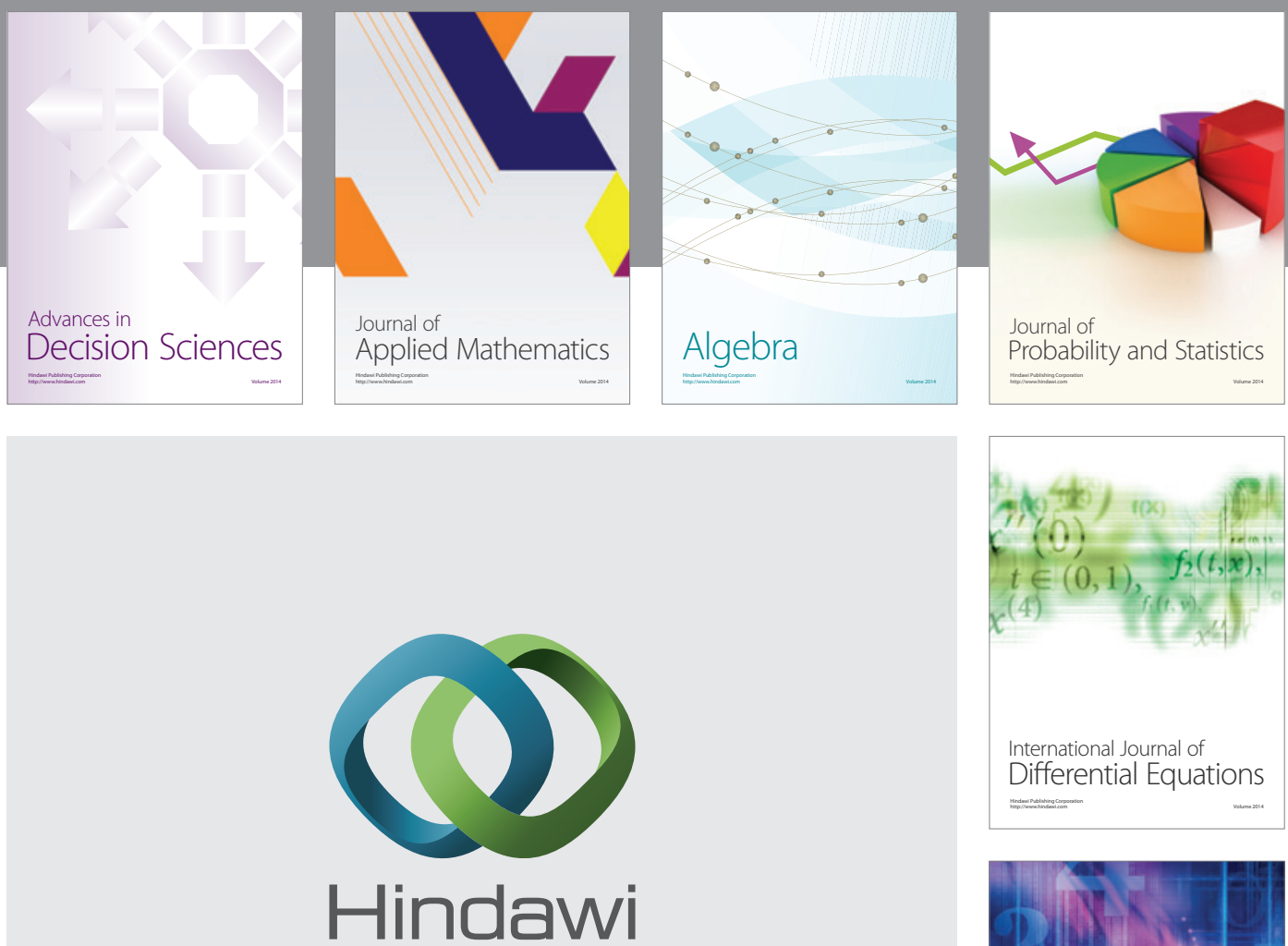

Submit your manuscripts at http://www.hindawi.com
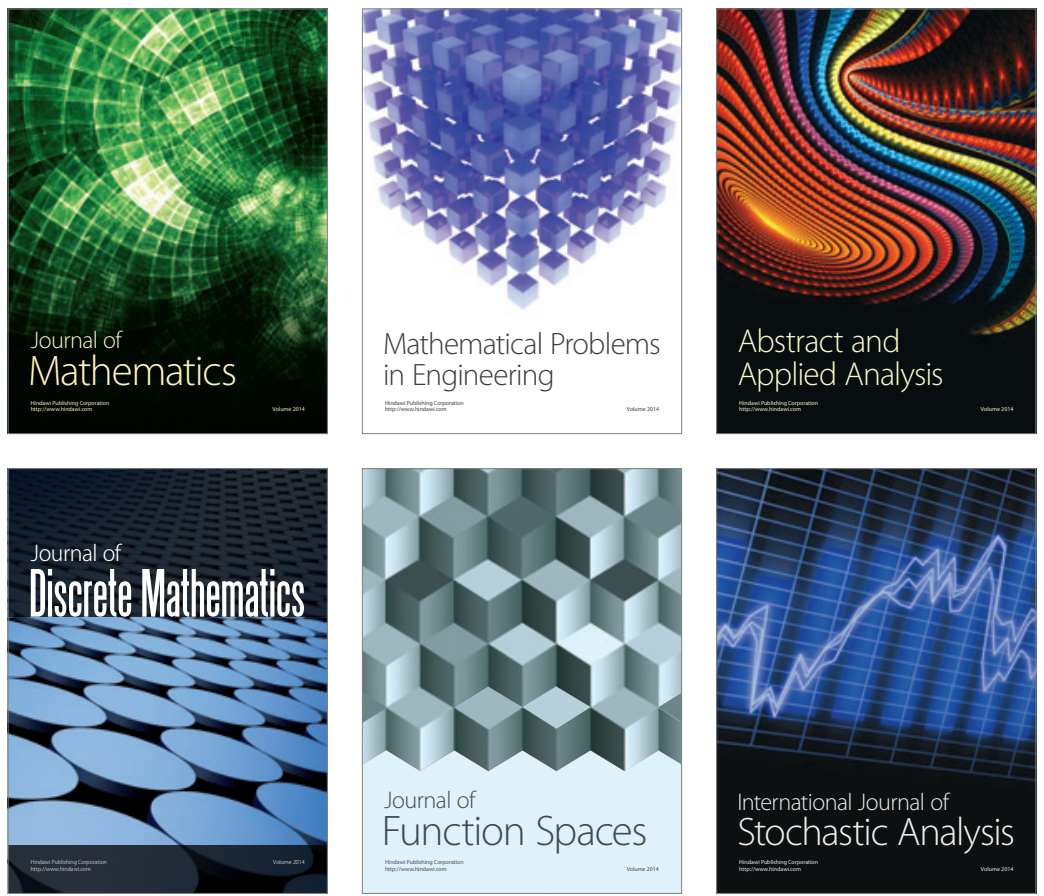

Journal of

Function Spaces

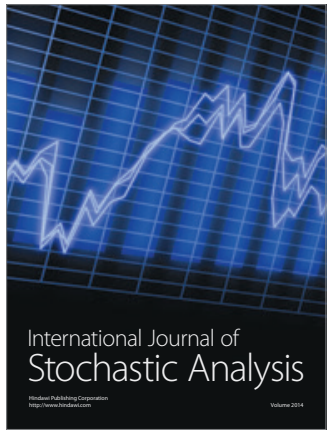

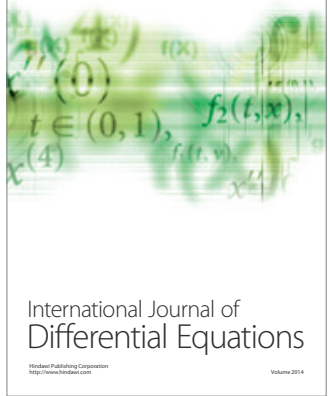
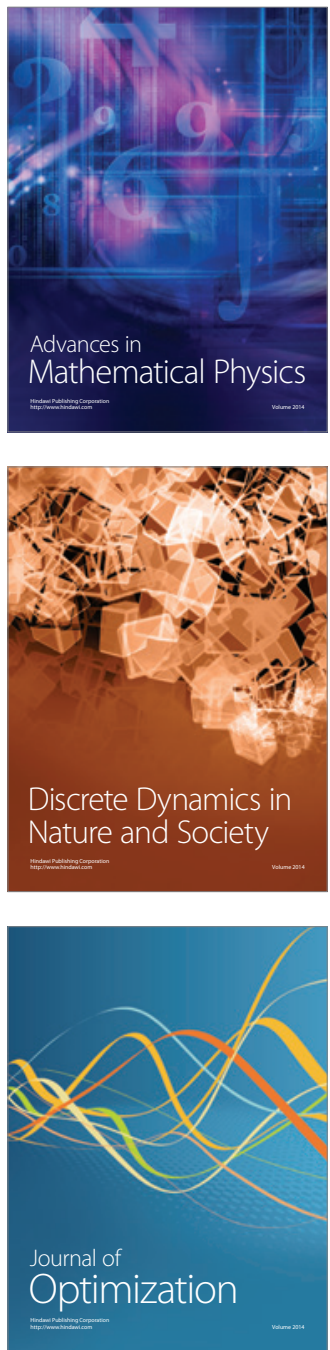